\title{
Liver cancer cells: targeting and prolonged-release drug carriers consisting of mesoporous silica nanoparticles and alginate microspheres
}

This article was published in the following Dove Press journal:

International Journal of Nanomedicine

5 June 2014

Number of times this article has been viewed

\author{
Yu-Te Liao' \\ Chia-Hung Liu² \\ Jiashing $\mathrm{Yu}^{\prime}$ \\ Kevin C-W Wu ${ }^{1,3}$ \\ 'Department of Chemical Engineering, \\ National Taiwan University, Taipei, \\ Taiwan; ${ }^{2}$ Department of Urology, \\ Taipei Medical University-Shuang Ho \\ Hospital, New Taipei City, Taiwan; \\ ${ }^{3}$ Division of Medical Engineering \\ Research, National Health Research \\ Institutes, Zhunan Township, Miaoli \\ County, Taiwan
}

\begin{abstract}
A new microsphere consisting of inorganic mesoporous silica nanoparticles (MSNs) and organic alginate (denoted as MSN@Alg) was successfully synthesized by air-dynamic atomization and applied to the intracellular drug delivery systems (DDS) of liver cancer cells with sustained release and specific targeting properties.MSN@Alg microspheres have the advantages of MSN and alginate, where MSN provides a large surface area for high drug loading and alginate provides excellent biocompatibility and $\mathrm{COOH}$ functionality for specific targeting. Rhodamine $6 \mathrm{G}$ was used as a model drug, and the sustained release behavior of the rhodamine 6G-loaded MSN@Alg microspheres can be prolonged up to 20 days. For targeting therapy, the anticancer drug doxorubicin was loaded into MSN@Alg microspheres, and the (lysine)4-tyrosine-arginine-glycine-aspartic acid ( $\mathrm{K}_{4}$ YRGD) peptide was functionalized onto the surface of MSN@Alg for targeting liver cancer cells, hepatocellular carcinoma (HepG2). The results of the 3-[4,5-dimethylthiazol-2-yl]-2,5 diphenyl tetrazolium bromide (MTT) assay and confocal laser scanning microscopy indicate that the MSN@Alg microspheres were successfully uptaken by HepG2 without apparent cytotoxicity. In addition, the intracellular drug delivery efficiency was greatly enhanced (ie, 3.5-fold) for the arginine-glycine-aspartic acid (RGD)-labeled, doxorubicin-loaded MSN@Alg drug delivery system compared with the non-RGD case. The synthesized MSN@Alg microspheres show great potential as drug vehicles with high biocompatibility, sustained release, and targeting features for future intracellular DDS.
\end{abstract}

Keywords: alginate, mesoporous silica nanoparticles, atomization, sustained release, targeting therapy

\section{Introduction}

Hepatocellular carcinoma (HCC) predicted at least 25,000 newly diagnosed cases also caused near 20,000 patients died in United States in 2012. HCC is a common, malignant disease with a great capability for chemoresistance. ${ }^{1}$ Not only a threat in developed countries, HCC also affects people in developing countries. ${ }^{2,3} \mathrm{P}$-glycoprotein $(\mathrm{P}-\mathrm{gp})$ is a well-known protein that causes problems in chemotherapy. ${ }^{4} \mathrm{P}$-gp proteins, which have been found in most human HCC tissues, are capable of extruding various positively charged xenobiotics, such as doxorubicin (DOX), which is commonly used as chemotherapy for HCC. ${ }^{5}$ To overcome chemoresistance, the drug would be modified with P-gp inhibitors or loaded into drug carriers such as microspheres, liposomes, or nanoparticles. ${ }^{6-8}$

The synthesis of a drug delivery carrier is important for increasing the drug loading capacity, controlling the release kinetics of the loaded drugs, and targeting the position of cancers. ${ }^{9-11}$ Among these features, drug carriers exhibiting simultaneous sustained
Correspondence: Kevin C-W Wu Department of Chemical Engineering, National Taiwan University, No I, Sec 4, Roosevelt Road, Taipei 10617, Taiwan Tel +886233663064

Email kevinwu@ntu.edu.tw 
release and cancer cell targeting are especially in demand. Sustained release means the loaded drug is released from the carrier slowly, which keeps its concentration higher than the effective concentration for a longer time, which means its circulation time can be improved greatly. ${ }^{12,13}$ Specific targeting means the drug can be specifically delivered to tumors and thus can avoid the distribution of carriers to pathological cells, minimizing the adverse effects of drug on healthy cells. ${ }^{14,15}$ Porous materials with a morphology of particles and controllable sizes have proven useful for high loading of therapeutic drugs, protection from environmental interference, control of release behaviors, and transportion to target sites. ${ }^{16-18}$

Novel technologies that combine a chemical synthesis approach with a physical engineering process have created numerous promising drug delivery carriers. For example, mesoporous materials have been widely synthesized through a chemical sol-gel method in the presence of structure-directing agents. ${ }^{19-22}$ The synthesized mesoporous materials exhibit several advantages such as high surface area, ordered structure, controllable pore size, and various surface functionalities, which make these materials useful in biomedical applications, especially in drug delivery systems. $^{23}$

In contrast, a natural polysaccharide, called alginate, is recognized as the most abundant marine biopolymer in the world and is known for its high biocompatibility and biodegradability. ${ }^{24-26}$ Alginate has been used in the food industry because it is generally regarded as safe by the US Food and Drug Administration (GRN 328). In general, alginate can chelate with most of divalent cations to form a rigid structure, ${ }^{27}$ and the structure of alginate could be made in many different morphologies such as gel, ${ }^{28}$ particle, ${ }^{29}$ fiber, ${ }^{30}$ bulk, ${ }^{31}$ and film. ${ }^{32}$ The unique cation-induced gelation properties of alginate enable the encapsulation of any nanomaterials with enhanced loading. In terms of drug delivery, microparticles or microcapsules made of alginate have been used successfully. ${ }^{33-35}$ There are three common methods for synthesizing alginate carriers: coacervation, ${ }^{36}$ layer by layer, ${ }^{37}$ and atomization. ${ }^{38}$

Liang et al loaded paclitaxel, which was a strong hydrophobic drug, into poly ( $\gamma$-glutamic acid)-poly(lactide) nanoparticles functionalized with galactosamine (P/Gal-NPs) as a new targeted drug delivery system to treat HepG2. ${ }^{39}$ The results showed that receptor-mediated endocytosis increased the efficiency of paclitaxel compared with clinically available paclitaxel formulation. To efficiently deliver DOX into hepatoma cells, Guo et al mixed glycyrrhetinic acid-modified alginate and DOX-modified alginate together (GA-ALG/ DOX-ALG NPs). ${ }^{40}$ Compared with DOX and DOX-ALG NPs, the inhibiting rate of tumor growth after treatment with GA-ALG/DOX-ALG NPs increased about 1.6- and 1.3-fold. More important, no mice died after being treated with GAALG/DOX-ALG NPs (40\% of mice died after treatment with DOX). Iancu et al also distributed a targeting delivery system for thermal ablation. ${ }^{41}$ They functionalized human serum albumin on multiwalled carbon nanotubes to target the Gp60 receptor existing on HepG2. After being irradiated with a $2 \mathrm{~W}, 808 \mathrm{~nm}$ laser beam, the apoptotic rate of HepG2 ranged from $88.24 \%$ at the first 60 seconds to $92.34 \%$ at 30 minutes. In contrast, lower necrotic rates from human hepatocytes were obtained after treatment with HSA-multiwalled, human serum albumin in a similar manner.

We have previously adopted the advantages of both mesoporous materials and the cation-induced gelation property of alginate to fabricate mesoporous silica nanoparticle (MSN)-encapsulated alginate microspheres (called MSN@ Alg) by atomization as a new effective drug delivery carrier. ${ }^{42}$ Here we extend the application of the MSN@Alg for specific targeting therapy (Figure 1). Because of the high surface area of MSN, various cargos (ie, a fluorescent dye [Rhodamine $6 \mathrm{G}(\mathrm{R} 6 \mathrm{G})$ ] or a therapeutic drug [DOX]) could be loaded effectively. The cargo-loaded MSN materials were then introduced into alginate on gelation, and the microspherical morphology could be formed after optimizing the reaction conditions of atomization. The fabricated MSN@ Alg microspheres exhibit a prolonged release property. More important, the abundant carboxylic groups of alginate afford further functionalization of MSN@Alg with cancer cell-targeting peptides (ie, $\mathrm{K}_{4}$ YRGD). ${ }^{43}$ The DOX-loaded, K YRGD-functionalized MSN@Alg microspheres have been proven to exhibit enhanced efficiency of intracellular drug delivery to human liver cancer cells (HepG2).

\section{Materials and methods Chemicals}

Phosphate buffered saline (PBS), fetal bovine serum (FBS), and Modified Eagle's Medium (MEM) were purchased from Biological Industries Israel Beit-Haemek Ltd. (Kibbutz Beit-Haemek, Israel). R6G, fluorescein isothiocyanate (FITC), DOX hydrochloride, Brij-97, cetyltrimethylammonium bromide $\left[\mathrm{C}_{16} \mathrm{H}_{33} \mathrm{~N}\left(\mathrm{CH}_{3}\right)_{3} \mathrm{Br}\right]$, $N$-(3-dimethylaminopropyl)- $N$ '-ethylcarbodiimide hydrochloride (EDC), N-hydroxysuccinimide (NHS), 3-aminopropyltrimethoxysilane (97\%), tetraethoxysilane, and dimethylphthalate $(>99 \%)$ were purchased from Sigma-Aldrich 

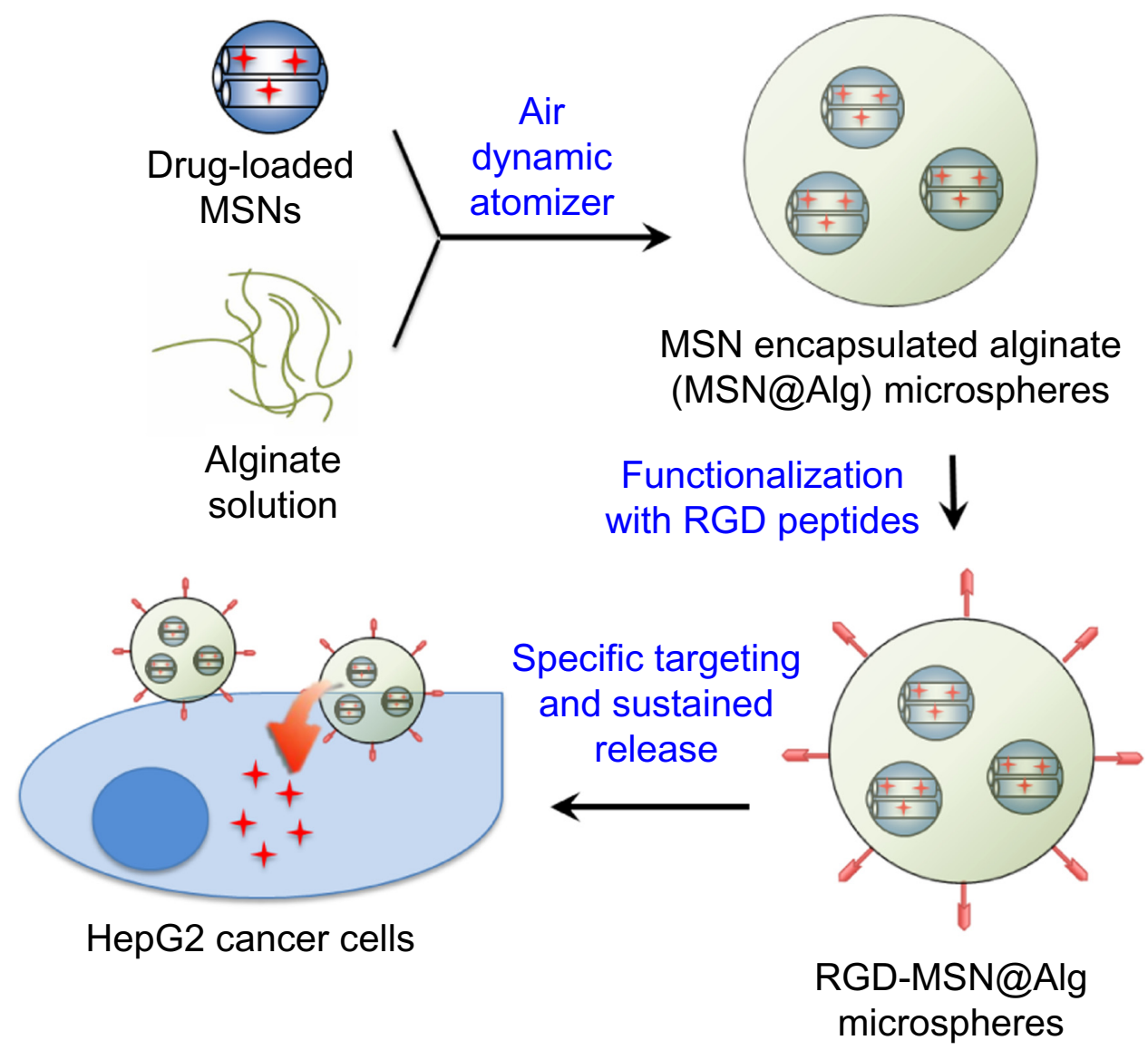

Figure I Illustration representing the drug-adsorbed mesoporous silica nanoparticles (doxorubicin-MSN) encapsulated into RGD-containing peptide functionalized alginate microspheres.

Abbreviations: MSNs, mesoporous silica nanoparticles; MSN@Alg, MSNs and organic alginate; RGD, arginine-glycine-aspartic acid.

(St Louis, MO, USA). The arginine-glycine-aspartic acid (RGD)-based peptide $\mathrm{K}_{4}$ YRGD, was purchased from Kelowna International Scientific Inc (Kelowna, Taiwan).

\section{Synthesis of MSNs}

MSNs were synthesized as in our previous report. ${ }^{44}$ Briefly, $6.92 \mathrm{~mL}$ Brij-97 was added into $180 \mathrm{~mL}$ deionized water and stirred for 1 hour at room temperature. After Brij-97 was dissolved completely, $0.3 \mathrm{~mL} 3$-aminopropyltrimethoxysilane and $0.08 \mathrm{~mL}$ DOP were added to the Brij-97 aqueous solution. After stirring for 30 minutes, $6.7 \mathrm{~mL}$ tetraethoxysilane was then added. After stirring for 24 hours at room temperature, the mixed solution was then refluxed at $100^{\circ} \mathrm{C}$ for another 24 hours. The white precipitate collected by filtration was washed with methanol several times to remove the Brij-97. Finally, the sample was collected and dried in vacuum.

\section{Synthesis of MSN@Alg microspheres}

MSN@Alg microspheres were fabricated with an airdynamical atomizer (VAR J30; Nisco, Zurich, Switzerland) with optimized conditions. First, the MSN sample (0.3 wt\%) was suspended into an alginate aqueous solution ( $1 \mathrm{wt} \%)$, and the whole mixture was transferred into a syringe. The MSNcontaining alginate solution was extruded out by a syringe pump equipped with an air nozzle and then was dropped into another $\mathrm{Ca}^{2+}$-containing solution $(0.1 \mathrm{M})$. Alginate started cross-linking and formed microspheres when it was reacted with $\mathrm{Ca}^{2+}$. For the formation of a spherical morphology, several parameters were optimized: diameter of nozzle, $0.35 \mathrm{~mm}$; flow rate of solution, $0.5 \mathrm{~mL} /$ minute; pressure, 500 mbar; and distance from nozzle to solution, $6 \mathrm{~cm}$.

\section{Functionalization of MSN@Alg microspheres with targeting ligands}

Because there are carboxyl groups on the alginate and amine groups on the peptide $\mathrm{K}_{4}$ YRGD, we used EDC/NHS chemistry to graft the peptide on alginate. $\operatorname{EDC}(0.27 \mathrm{mM})$ and NHS (0.14 mM) were dissolved into PBS (10 mM pH 7.4), and alginate was then added into the PBS solution, stirring for 1 hour at $4^{\circ} \mathrm{C}$. After that, $\mathrm{K}_{4}$ YRGD $(12 \mu \mathrm{g})$ was added 
into solution, stirring for 4 hours at $4^{\circ} \mathrm{C}$. Finally, MSN was suspended into the $\mathrm{K}_{4}$ YRGD-functionalized alginate solution, and the mixture was used for the formation of microspheres by atomization, as described earlier.

\section{Functionalization of MSN with fluorescein isothiocyanate}

Same molar of fluorescein isothiocyanate (FITC) was stirred with (3-Aminopropyl)triethoxysilane (APTES) in methanol for 1 hour to form FITC-APTES. One hundred milligrams of MSN were grafted with FITC-APTES in toluene at $110^{\circ} \mathrm{C}$ for 24 hours. After grafted with FITCAPTES, FITC-MSN was washed with methanol several times to remove ungrafted FITC-APTES. The sample was used to synthesize FITC-MSN-encapsulated alginate microspheres after being dried in lyophilizer.

\section{Characterization}

The morphology of MSN and alginate was observed using a scanning electron microscope (Nova ${ }^{\mathrm{TM}}$ Nano SEM; FEI, Hillsboro, OR, USA) and optical microscope (Nikon TE2000-U; Nikon Corporation, Tokyo, Japan), respectively. The porous properties of MSN were examined with nitrogen adsorption-desorption (ASAP2010; Micromeritics Instrument Corporation, Norcross, GA, USA). The distribution of MSN inside of alginate beads was observed with a confocal laser scanning microscope (Leica TCS SP5 II; Leica Microsystems, Wetzlar, Germany). To make sure DOX/ MSN@Alg was indeed functionalized with targeting ligands (ie, peptide), samples were examined with Fourier transform infrared spectroscopy (Spectrum 100; PerkinElmer, Waltham, MA, USA) and Ultraviolet-visible (UV-Vis) spectrometry (V-670; JASCO, Easton, MD, USA). Samples used for Fourier transform infrared spectroscopy measurements were prepared by mixing the vacuum-dried samples with potassium bromide $(\mathrm{KBr})(\mathrm{KBr}$ :sample =100:1). The mixture was then ground extensively and pressed into a translucent disc.

\section{Loading and in vitro release of cargos}

Two kinds of cargos were used for the loading and release tests in this study. R6G was chosen as a model drug to study the release behavior, and DOX was chosen as a chemotherapy drug for intracellular drug delivery. For loading guest molecules, MSN (30 mg) was suspended in $10 \mathrm{~mL}$ guest molecules containing an aqueous solution $\left(10^{-5} \mathrm{M}\right.$ for R6G and $10^{-4} \mathrm{M}$ for DOX), and the mixture was stirred for 24 hours in room temperature. For DOX/MSN, $30 \mathrm{mg}$ MSN was suspended into $10 \mathrm{~mL}$ of aqueous solution and stirred for 24 hours. The cargo-loaded MSN was collected by centrifuge and dried in vacuum. For the preparation of cargo-loaded MSN@Alg microspheres, the cargo-loaded MSN was resuspended in the alginate solution, and this solution was dropped into a $\mathrm{Ca}^{2+}$-containing solution by the air-dynamic atomizer with optimized reaction conditions, as described earlier.

The fluorescence of the supernatant was measured by photoluminescence (F-7000 fluorescence spectrophotometer; Hitachi Ltd, Tokyo, Japan) to quantify the amount of R6G in the supernatant $\left(\lambda_{\mathrm{ex}}, 526 \mathrm{~nm} ; \lambda_{\mathrm{em}}, 551 \mathrm{~nm}\right)$. The loading efficiency and capacity were calculated according to the following equations:

Encapsulation efficiency (\%)

$=\frac{\text { Drug input }(\mathrm{mg})-\text { Drug in supernatant }(\mathrm{mg})}{\text { Drug input }(\mathrm{mg})} \times 100 \%$,

and

Loading capacity $(\mathrm{mg} / \mathrm{g})$

$$
=\frac{\text { Drug input }(\mathrm{mg})-\text { Drug in supernatant }(\mathrm{mg})}{\text { Drug-loaded material }(\mathrm{g})} \text {. }
$$

For the in vitro release of R6G molecules, R6G-loaded samples (10 mg for R6G/MSN and 43 mg for R6G/MSN@ Alg) were added into PBS ( $\mathrm{pH} 7.4$ ) solutions with various volumes (10-30 $\mathrm{mL})$ and concentrations (10-50 mM), without stirring, at $37^{\circ} \mathrm{C}$. At predetermined time intervals, the mixture was centrifuged (13,000 rpm, 30 minute), and $0.4 \mathrm{~mL}$ supernatant was extracted and replaced with fresh buffers. R6G in the supernatant was quantified by measuring its fluorescence (F-7000 fluorescence spectrophotometer).

\section{Cell culture and MTT assay}

Hepatic cancer cells (HepG2) and fibroblasts (3T3) were purchased from the National Health Research Institutes in Taiwan and were routinely cultured in MEM containing 10\% FBS and incubated at $37^{\circ} \mathrm{C}$ in a humidified atmosphere with $5 \%$ $\mathrm{CO}_{2}$. Cell viability was measured by 3-[4,5-dimethylthiazol2-yl]-2,5 diphenyl tetrazolium bromide (MTT) assay. Cells were cultured in 24-well microplates $\left(10^{5}\right.$ cells/well) with $0.5 \mathrm{~mL}$ MEM containing 10\% FBS. After 1 day, the medium was replaced with medium without FBS and cultured for another day. Samples including free DOX molecules, MSN@ Alg, DOX/MSN@Alg, and DOX/MSN@Alg-RGD with various concentrations were suspended into serum-free MEM media. The media were then added to each well, and the cells were incubated at $37^{\circ} \mathrm{C}$ for 24 hours. The medium was then removed, and the wells were washed twice with $1 \mathrm{~mL}$ PBS. To 
each well, $0.5 \mathrm{~mL}$ MTT solution $(0.5 \mathrm{mg} / \mathrm{mL}$ in the medium) was added, and the cells were incubated for an additional 4 hours. The medium was then replaced with $0.5 \mathrm{~mL}$ dimethyl sulfoxide. The plates were left stationary for 4 hours to dissolve the blue crystals, and the absorbance was recorded by a microplate reader at a wavelength of $570 \mathrm{~nm}$.

To quantify the amount of MSN uptaken by cells, the sample-uptaken cells were cultured in 6 -well plates $\left(2 \times 10^{5} /\right.$ well $)$ for 4 hours. After that, trypan blue was added to the plates for 10 minutes to quench the fluorescence of FITC outside of the cells. Then, the lysis buffer was added to disrupt the cell membrane and the medium was analyzed with photoluminescence spectroscopy. To exclude possible receptor-mediated endocytosis, HepG2 was cultured in sucrose-containing medium (0.45 M) for $10 \mathrm{~min}$ before the addition of samples.

\section{Confocal laser scanning microscopy}

HepG2 cells were seeded onto 4-well Lab-Tek slides at a density of $2.5 \times 10^{4}$ cells per well. After incubation at $37^{\circ} \mathrm{C}$ with $5 \% \mathrm{CO}_{2}$ overnight, the medium was removed and the slides were washed twice with PBS. To trace the position of samples inside the cells, MSN was previously labeled with a fluorescent dye FITC. FITC-labeled MSN was then encapsulated into alginate to form FITC-MSN@Alg and FITC-MSM@Alg-RGD. To each well, $0.5 \mathrm{~mL}$ PBS (10\% FBS) containing samples $(100 \mu \mathrm{g} / \mathrm{mL})$ was added. After incubation for an additional 4 hours, the supernatant was removed and the slides were washed extensively with PBS. To each well, $0.5 \mathrm{~mL}$ PBS containing $28 \mu \mathrm{M}$ 4',6-diamidino-2phenylindole (DAPI) was added to stain the nuclei for 20 minutes.
Then, the slides were washed 3 times with PBS and covered with coverslips. Confocal laser scanning microscopy was performed on a Leica TCS SP5 II equipped with a $63 \times$ oil immersion objective lens. Blue fluorescence was produced in the DAPI-stained nuclei by exciting the cells with an ultraviolet laser $\left(\lambda_{\text {ex }}, 405 \mathrm{~nm}\right)$. The green fluorescence emitted from the FITC-labeled MSN@ Alg was visualized by excitation at $488 \mathrm{~nm}$.

\section{Results and discussion \\ Synthesis and characterization of MSN@Alg microspheres}

As shown in Figure 2a-1-and 2a-2, the MSN material exhibited a typical spherical morphology with a diameter around $500 \mathrm{~nm}$ and a bulk-like morphology with a diameter around $1 \mu \mathrm{m}$. The corresponding BET (Brunauer-Emmett-Teller)-specific surface area and BJH (Barret-Joyner-Halenda) pore size of the MSN was calculated as $285 \mathrm{~m}^{2} / \mathrm{g}$ and $5 \mathrm{~nm}$ and $30 \mathrm{~nm}$, respectively, from $\mathrm{N}_{2}$ adsorption-desorption isotherm (Figure $\mathrm{S} 1$ ). The large pore size of the MSN indicates a potential adsorption of large macromolecular drugs such as proteins, genes, and si-RNA. As shown in an optical microscopic image (Figure $2 b)$, the alginate microspheres with a diameter around 20-30 $\mu \mathrm{m}$ were also successfully fabricated after we optimized the operating conditions of atomization. It has been reported that three factors (concentration of alginate, pressure of air flow, and flow rate of solution) are critical for formation of alginate microspheres. ${ }^{45}$ Here, in addition to these three factors, we optimized two more factors: the distance between the cross-linker (ie, $\mathrm{Ca}^{2+}$-containing) solution and nozzle and the
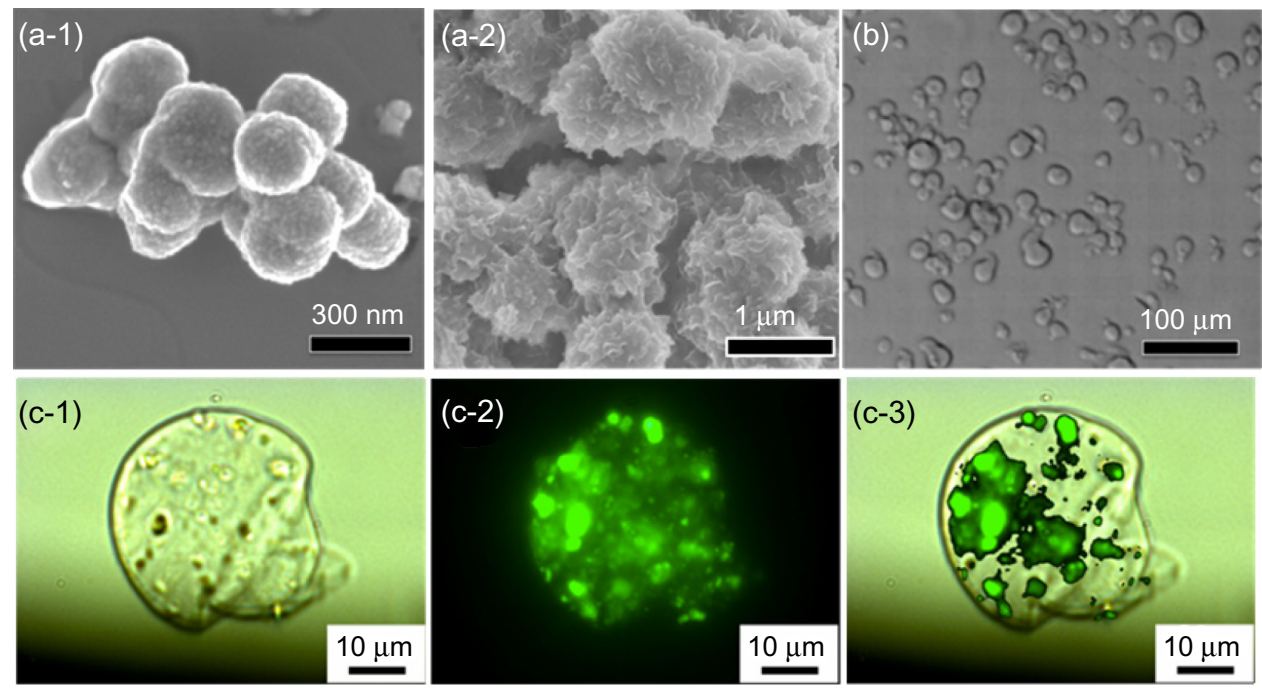

Figure 2 (a) Typical scanning electron microscopy image of mesoporous silica nanoparticles (MSNs) used in this study, with (a-I) spherical morphology and (a-2) bulk-like morphology. (b) A typical optical microscopy image of alginate microspheres. (c) Fluorescent images of a fluorescein isothiocyanate-MSN-encapsulated alginate microsphere: (c-I) DIC image, (c-2) green fluorescent image from fluorescein isothiocyanate-adsorbed MSNs, and (c-3) merged image.

Abbreviation: DIC, differential interference contrast microscopy. 

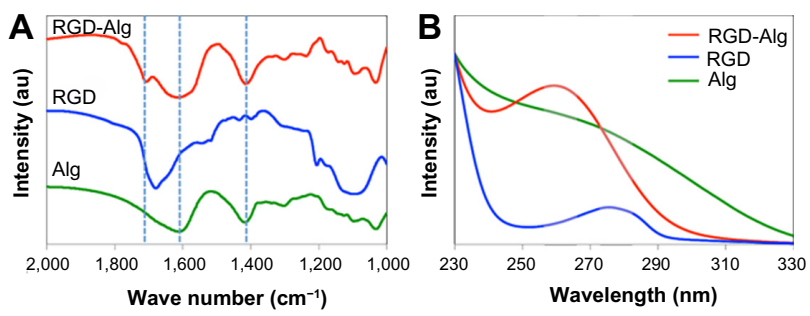

Figure 3 (A) Fourier transform infrared spectroscopy and (B) UV-Vis spectroscopy of samples including pure alginate microspheres, pure $\mathrm{K}_{4} Y R_{\mathrm{R}} \mathrm{GD}$ peptides, and $\mathrm{K}_{4}$ YRGD peptide-functionalized alginate microspheres. Alg represents alginate. Abbreviations: Alg, alginate microparticle; UV-Vis, ultraviolet-visible; RGD, arginine-glycine-aspartic acid.

rotation speed of the cross-linker solution to obtain a spherical shape. The final conditions were optimized as follows: $2 \mathrm{wt} \%$ for concentration of alginate, $500 \mathrm{mbar}$ for pressure, $0.5 \mathrm{~mL} /$ minute for flow rate of solution, $6 \mathrm{~cm}$ for the distance from cross-linker solution to nozzle, and $300 \mathrm{rpm}$ for the rotation speed of the cross-linker solution.

To confirm successful encapsulation and directly visualize the location of MSN inside alginate microspheres, we previously linked a green fluorescent dye FITC onto the surface of MSN. As shown in Figure 2c, a single alginate microsphere was observed under transmission mode (Figure 2c-1), and many green spots that represented the FITC-labeled MSN materials could be clearly seen under fluorescence mode (Figure 2c-2), indicating a homogeneous distribution. When the two images were merged (Figure 2c-3), it showed that the FITC-labeled MSN materials were encapsulated in the alginate microspheres.

For specific targeting of the cancer cell with the $\alpha_{\mathrm{v}} \beta_{3}$ receptor, one of the RGD-containing peptides, $\mathrm{K}_{4} \mathrm{YRGD}$, was applied and labeled on the external surface of MSN@Alg microspheres. The molar ratio of alginate to EDC to peptide used in this study was 1,000:5:2. The successful addition of the $\mathrm{K}_{4} \mathrm{YRGD}$ peptide was confirmed by Fourier transform infrared spectroscopy and UV-Vis spectroscopy. As shown in Figure 3A, two bands at around $1,400 \mathrm{~cm}^{-1}$ and $1,600 \mathrm{~cm}^{-2}$ were attributed to the $\mathrm{C}-\mathrm{O}$ symmetrical and asymmetrical stretching, respectively, of the $\mathrm{COOH}$ group in alginate. ${ }^{46}$ The band at $1,680 \mathrm{~cm}^{-1}$ represents amine I from the lysine of the RGD peptide. ${ }^{47}$ This band shifted slightly to a higher wavelength when the $\mathrm{K}_{4}$ YRGD peptide was linked to MSN@Alg microspheres. In addition, the intensity of the band was weak because of the low molar ratio of $\mathrm{K}_{4} \mathrm{YRGD}$ to alginate (ie, 1:500). Because the $\mathrm{K}_{4}$ YRGD peptide contains tyrosine, which means there will be absorption in the ultraviolet range, we further applied UV-Vis spectroscopy to approve the existence of the $\mathrm{K}_{4} \mathrm{YRGD}$ peptide on MSN@Alg microspheres. As shown in Figure 3B, pure $\mathrm{K}_{4}$ YRGD peptide showed an adsorption at $275 \mathrm{~nm}$. After this peptide was labeled on MSN@Alg, the absorption peak was shifted to $260 \mathrm{~nm}$. In contrast, there was no absorption peak on either $275 \mathrm{~nm}$ or $260 \mathrm{~nm}$ for pure alginate. These results demonstrate that the $\mathrm{K}_{4}$ YRGD peptide was labeled on the alginate of MSN@Alg microspheres successfully.

\section{Loading and sustained release of guest molecules}

The maximum adsorption amounts of R6G and DOX in MSN were calculated to be $3.1 \times 10^{-3} \mu \mathrm{mol} / \mathrm{mg}$ and $4.7 \times 10^{-3} \mu \mathrm{mol} / \mathrm{mg}$, respectively, where the corresponding loading efficacy was $29 \%$ and $4 \%$, respectively. The low loading efficacy for DOX was a result of the hydrophobicity of DOX; thus, it is difficult for hydrophobic DOX molecules to be adsorbed on the surface of hydrophilic MSN.

Next, the R6G- or DOX-loaded MSN materials were encapsulated into alginate microspheres by atomization, and the release behavior of R6G molecules was studied at different reaction conditions. In a PBS solution $(10 \mathrm{mM}, 10 \mathrm{~mL})$, when the concentration of the alginate increased from $0 \%$ (no alginate) to $0.3 \%$, the release of R6G molecules was greatly inhibited, as shown in Figure 4A. When the concentration further increased to $1 \%$, the release percentage was decreased to less than 5\%, even after 48 hours. The release behaviors were similar when the concentration exceeded
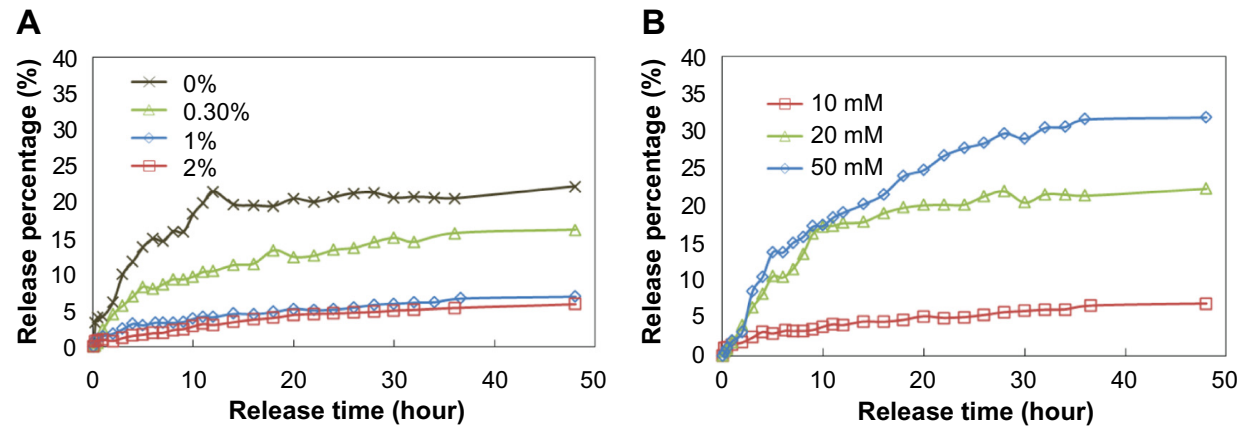

Figure 4 Release profiles of Rhodamine 6G-adsorbed mesoporous silica nanoparticle-encapsulated alginate microspheres in (A) different concentrations of alginate and (B) different concentrations of phosphate-buffered saline. 
$1 \%$. These results indicated that the R6G molecules were more difficult to release to the solution when there were more alginate layers outside of the R6G-loaded MSN. Although the release amount decreased as the amount of alginate increased, the release behavior of R6G without or with the presence of alginate (in other words, release from MSN or release from MSN@Alg, respectively) differed. Without alginate, R6G molecules could directly diffuse from the pores of MSN to solution, leading to a typical pseudo-first-order release profile. ${ }^{12}$ However, with the pres-

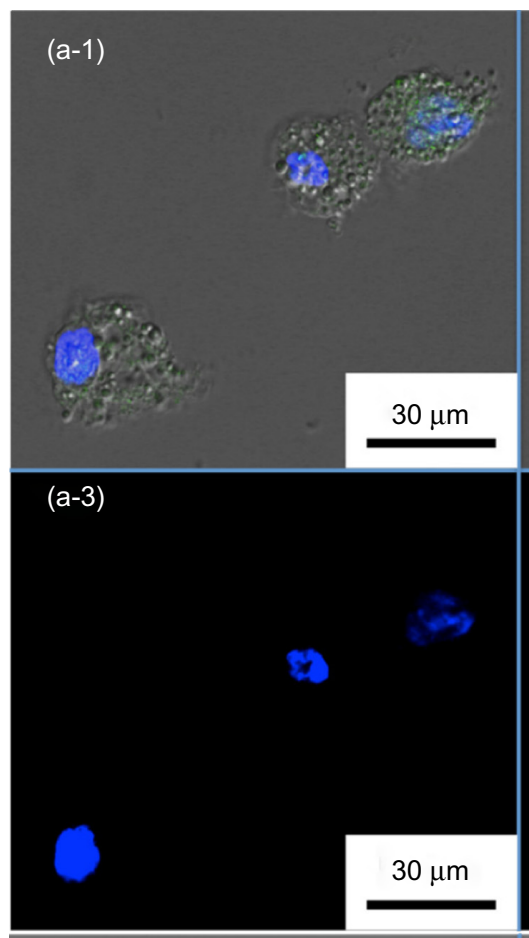

$(a-2)$

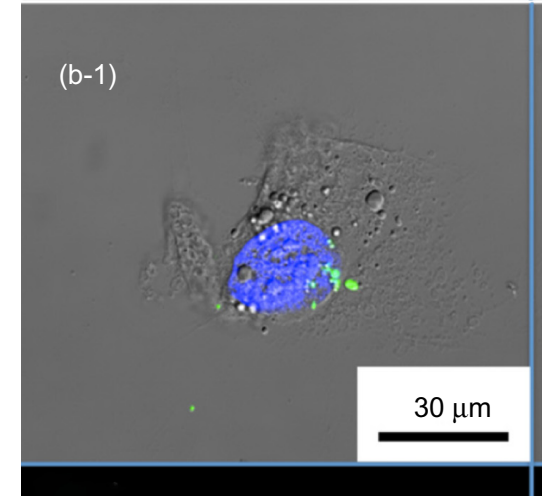

$(b-2)$
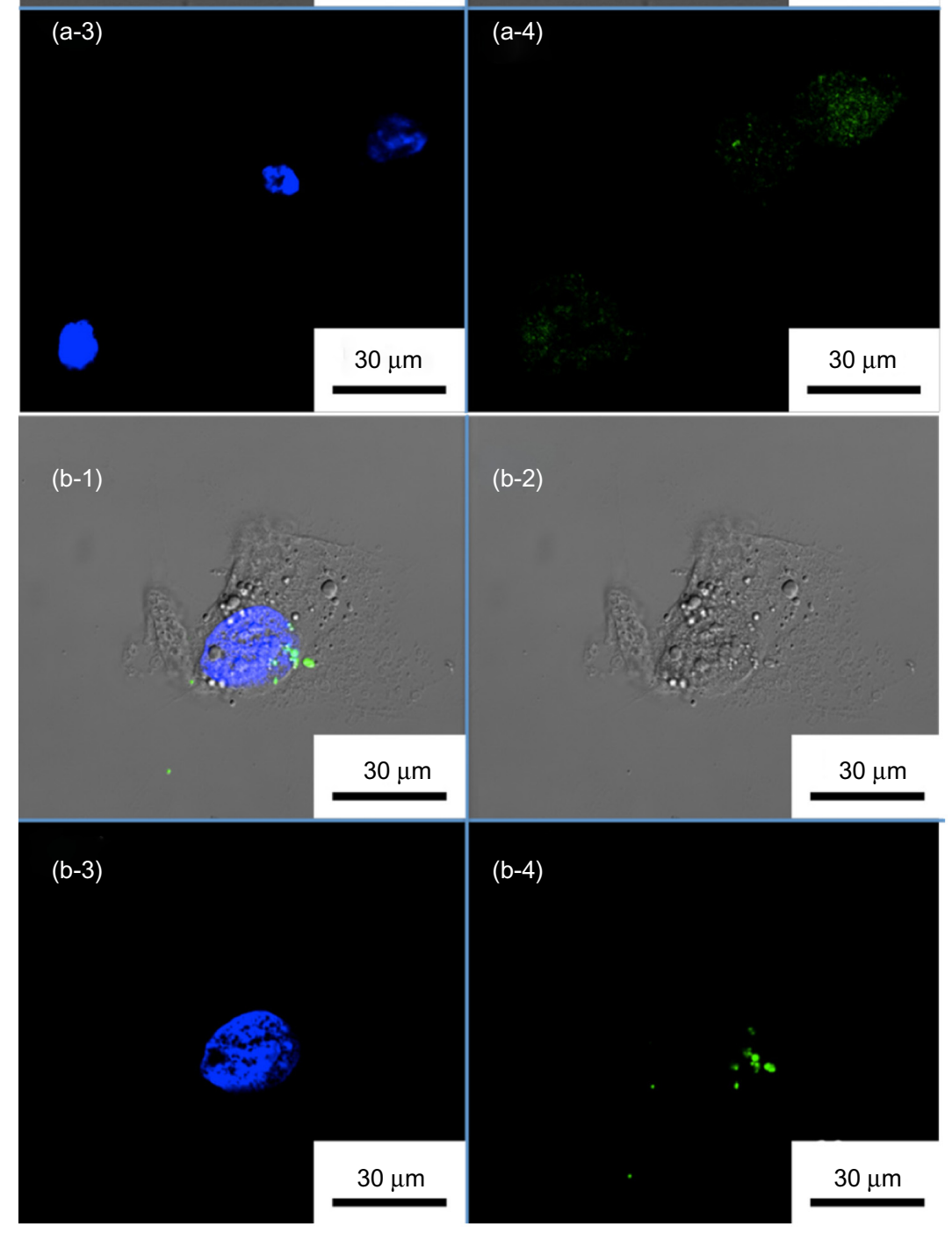

$(b-4)$

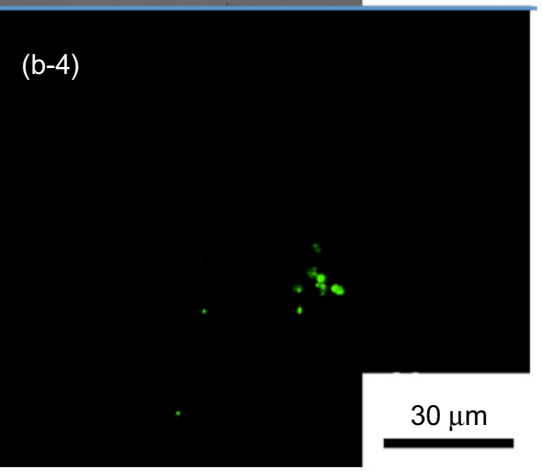

Figure 5 Confocal microscopy images of liver cancer cells (HepG2) treated with fluorescein isothiocyanate-labeled mesoporous silica nanoparticle-encapsulated alginate microspheres (a) with and (b) without $\mathrm{K}_{4}$ YRGD peptides. (a-I and $\mathbf{b}-\mathbf{-}$ ) Merged images. (a-2 and b-2) DIC images. (a-3 and b-3) Blue fluorescent images from DAPI-stained nuclei. (a-4 and b-4) Green fluorescent images from fluorescein isothiocyanate-labeled MSNs.

Abbreviations: DAPI, 4',6-diamidino-2-phenylindole; DIC, differential interference contrast microscopy; MSNs, mesoporous silica nanoparticles. 
ence of alginate, the release behavior of the R6G molecules changed to first order, and such linearly sustained release could be maintained for 40 days. Joshi et $\mathrm{al}^{35}$ reported similar gelatin@Alg microparticles via an air-dynamic atomizer. They found that the burst release of gelatin was $25 \%$ and that the release stopped after 5 days without the presence of alginate. However, the burst release decreased to $17 \%$, and the release was prolonged to 10 days, in the presence of alginate. Their study also confirmed that the encapsulation of guest molecules into alginate results in linear and sustained release.

Because the concentration of phosphate in a PBS solution would affect the binding between $\mathrm{Ca}^{2+}$ and alginate, and thus affect the release behavior of R6G from MSN@Alg, we studied the effect of PBS concentrations on the release behavior of R6G. Separately, 1wt\% R6G-loaded MSN@Alg samples were added into $10 \mathrm{~mL}$ PBS in different concentrations (ie, 10, 20, and $50 \mathrm{mM}$ ). As shown in Figure 4B, as the concentration of PBS increased from 10 to $50 \mathrm{mM}$, the release percentage of R6G increased from $8 \%$ to $32 \%$, respectively, after 48 hours. When there were more phosphate ions (higher PBS concentration), phosphate ions would interfere in the binding between $\mathrm{Ca}^{2+}$ and alginate, leading to a loose structure of alginate. Therefore, more R6G molecules could diffuse to solution. However, in all three cases, the release behaviors were attributed to the first-order release.

\section{Intracellular drug delivery}

To visualize the intracellular uptake of our MSN@Alg microspheres, and to demonstrate the enhanced cell uptake of specific targeting, FITC-labeled and $\mathrm{K}_{4}$ YRGD-functionalized MSN@Alg microspheres (denoted as FITC-MSN@, Alg-RGD) were prepared. As a reference, FITC-labeled MSN@Alg microspheres without grafting $\mathrm{K}_{4}$ YRGD peptides on the external surface were also prepared. Liver cancer cells (HepG2) exhibiting RGD receptors were used, and cells treated with FITC-labeled MSN@Alg with and without $\mathrm{K}_{4}$ YRGD peptides were observed through confocal laser scanning microscopy, as shown in Figure 5a and b. Cell morphologies were depicted in the differential interference contrast microscopy (DIC) image (Figure 5a-2 and 5b-2). Cells were stained with a blue fluorescent dye (4',6-diamidino-2-phenylindole DAPI) to specify the location of the nuclei under ultraviolet radiation (Figure 5a-3 and 5b-3). The FITC-labeled MSN@Alg samples emitted green fluorescence under an excitation wavelength of 488 nm (Figure 5a-4 and 5b-4). Because FITC molecules are not permeable to cells themselves, the green fluorescence could only derive from the FITC-labeled samples, indicating that the FITC-labeled MSN@Alg materials were indeed uptaken by HepG2 cells and localized in the cytoplasm. The morphologies of the HepG2 cells did not change after endocytosis of the microspheres (Figure 5a-1 and 5b-1), thereby suggesting little or negligible cytotoxicity of our samples. Image-Pro ${ }^{\circledR}$ (MediaCybernetics, Inc., Rockville, MD, USA) plus was used to quantify the amount of FITCMSN internalized into the cells. The results showed that the green area in one of the cells in Figure 5a-4 was 47,500 pixels in size, and the green area in the cell in Figure 5b-4 was 4,370 pixels. In contrast to Figure 5b-4, showing few green spots, Figure 5a-4 apparently showed more green spots, indicating that the existence of $\mathrm{K}_{4}$ YRGD peptide indeed promoted cell uptake.

In general, nanomaterials can be internalized into cells by clathrin-mediated endocytosis, also known as receptormediated endocytosis (RME). Several studies have been reported to show that RGD-containing nanomaterials could enhance the cell uptake through the RME pathway. ${ }^{48-50}$ To prove that more FITC-MSN@Alg-RGD samples were indeed uptaken by cells, we used lysis buffer to decompose the cell membrane and analyzed the amount of FITC-MSNs inside the HepG2 cells with photoluminescence. As shown in Figure 6A, the intensity of fluorescence from FITC-MSN@ Alg-RGD was enhanced about 1.6-fold compared with that of FITC-MSN@Alg. Because the fluorescence outside of the cell membrane and on the surface of the membrane was quenched by trypan blue,${ }^{51}$ the fluorescence intensity could only come from FITC-MSN inside the cells.

To further prove that FITC-MSN@Alg-RGD materials were taken up by the RME pathway, HepG2 cells were cultured in medium containing a high concentration of sucrose, which can inhibit the mechanism of RME. ${ }^{52}$ As shown in Figure 6B, when cells were cultured in medium with sucrose, the uptake amount of FITC-MSN@Alg-RGD was decreased greatly (around 75\%). This result clearly indicated
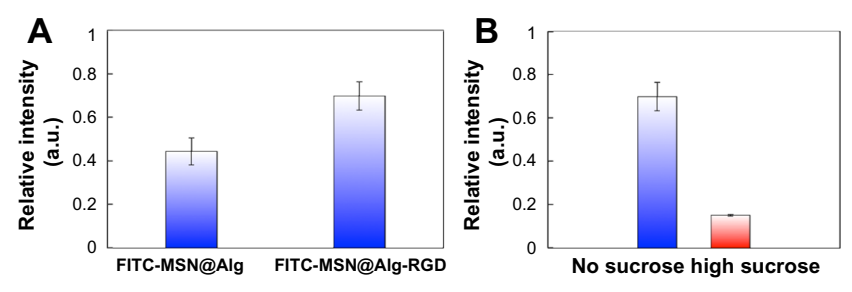

Figure 6 (A) Uptake amounts of samples with and without $K_{4}$ YRGD peptides in HepG2 cells. (B) Uptake amounts of fluorescein isothiocyanate-labeled mesoporous silica nanoparticle-encapsulated alginate with $\mathrm{K}_{4} \mathrm{YRGD}$ peptides with and without the presence of sucrose.

Abbreviations: FITC, fluorescein isothiocyanate; MSN@Alg, MSNs and organic alginate. 

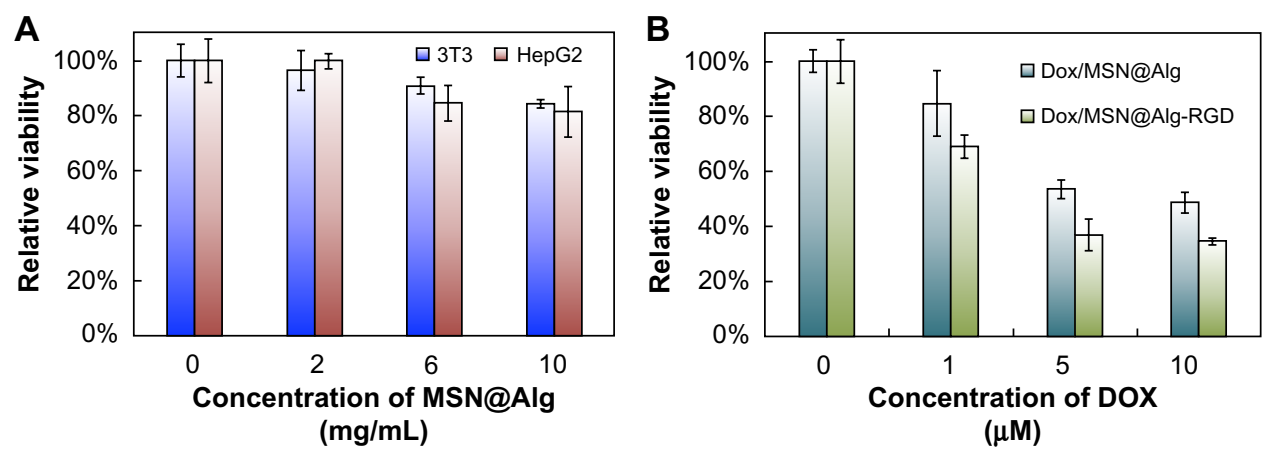

Figure 7 (A) MTT assays of normal cells (3T3) and cancer cells (HepG2) treated with fluorescein isothiocyanate-labeled mesoporous silica nanoparticle-encapsulated alginate (MSN@Alg) with different concentrations. (B) MTT assays of HepG2 cells treated with doxorubicin-loaded MSN@Alg microspheres with and without $\mathrm{K}_{4}$ YRGD peptides. Abbreviations: MTT, 3-[4,5-dimethylthiazol-2-yl]-2,5 diphenyl tetrazolium bromide; DOX, doxorubicin.

that the RGD peptide of the FITC-MSN@Alg-RGD could specifically target the $\alpha_{v} \beta_{3}$ receptor of HepG2 cells, and thus promoted cell uptake by the RME pathway.

Safety is of the utmost importance when new nanomaterials are used for biomedical applications. Although both alginate and MSN have been reported to exhibit high biocompatibility, the cytotoxicity of the newly synthesized materials in this study must be specified. MTT assay was used to quantify the effect of MSN@Alg microspheres on human liver cancer cells (HepG2) and fibroblast cells (3T3). As shown in Figure 7A, the fabricated MSN@Alg microspheres clearly exhibited excellent biocompatibility, and no adverse effects were observed up to a high concentration $(10,000 \mu \mathrm{g} / \mathrm{mL})$. The excellent biocompatibility of MSN@ Alg microspheres ensures its potential for use in biomedical applications such as drug delivery systems.

To investigate intracellular drug delivery by MSN@ Alg-based microspheres, we chose a chemotherapeutic drug, DOX, for HepG2 cells. The cell viability of HepG2 cells was evaluated by MTT assay after 24 hours, with different dosages of DOX-loaded MSN@Alg microspheres (with and without the targeting $\mathrm{K}_{4}$ YRGD peptides). The amount of DOX released from MSN@Alg was calculated from release profiles in Figure 4. We have previously calculated that the median lethal concentration $\left(\mathrm{LC}_{50}\right)$ of DOX to HepG2 after 24 hours was $15.37 \mu \mathrm{M}$. Figure 7B showed that the cell viability decreased when the amount of DOX released from MSN@ Alg increased. We calculated the $\mathrm{LC}_{50}$ value for DOX-MSN@ Alg to be $8.34 \mu \mathrm{M}$. This result indicated that the MSN@Alg microspheres could be an effective drug carrier to deliver DOX drugs into the cell cytoplasm and that the delivery efficiency was enhanced about 1.85 -fold. In addition, when we used MSN@Alg-RGD as the drug carrier, the $\mathrm{LC}_{50}$ value further decreased to $4.35 \mu \mathrm{M}$, indicating that the existence of $\mathrm{K}_{4}$ YRGD peptides indeed encouraged cell uptake through the RME pathway. The delivery efficiency was about 3.5-fold higher than for free DOX molecules. Such an RGD-assisted delivery system has also been reported by other groups. ${ }^{49}$

\section{Conclusion}

This study reports the fabrication of MSN-encapsulated alginate (MSN@Alg) microspheres through air-dynamic atomization. The synthesized MSN@Alg microspheres exhibit excellent biocompatibility, good drug loading capacity, sustained release feature, and enhanced drug release efficacy. Several guest molecules, such as organic dyes and therapeutic anticancer drugs, can be loaded efficiently into MSN and released through controllable kinetics. With the functionalization of cancer celltargeting $\mathrm{K}_{4}$ YRGD peptides, drug-loaded MSN@Alg-RGD exhibited enhanced intracellular delivery efficacy toward liver cancer cells (HepG2) through receptor-mediated endocytosis.The MSN@Alg microspheres here show significant potential as effective and visually observable transmembrane delivery carriers for the intracellular controlled release of cell membrane-impermeable drugs. Further development of this type of MSN@Alg material can lead to a new generation of nanodevices for biomedical applications.

\section{Acknowledgments}

This research was supported by the National Science Council of Taiwan (101-2628-E-002-015-MY3), National Taiwan University (102R7842 and 102R7740) and National Health Research Institute (03A1-BNMP14-014).

\section{Disclosure}

The authors report no conflicts of interest in this work.

\section{References}

1. Siegel R, DeSantis C, Virgo K, et al. Cancer treatment and survivorship statistics, 2012. CA Cancer J Clin. 2012;62(4):220-241. 
2. Marra M, Sordelli IM, Lombardi A, et al. Molecular targets and oxidative stress biomarkers in hepatocellular carcinoma: an overview. J Transl Med. 2011;9:171.

3. Caraglia M, Giuberti G, Marra M, et al. Oxidative stress and ERK1/2 phosphorylation as predictors of outcome in hepatocellular carcinoma patients treated with sorafenib plus octreotide LAR. Cell Death Dis. 2011;2:e150.

4. Ambudkar SV, Dey S, Hrycyna CA, Ramachandra M, Pastan I, Gottesman MM. Biochemical, cellular, and pharmacological aspects of the multidrug transporter. Annu Rev Pharmacol Toxicol. 1999;39: 361-398.

5. Brito AF, Abrantes AM, Pinto-Costa C, et al. Hepatocellular carcinoma and chemotherapy: the role of p53. Chemotherapy. 2012;58(5): 381-386.

6. Tapiero H, Mishal Z, Wioland M, Silber A, Fourcade A, Zwingelstein G. Changes in biophysical parameters and in phospholipid composition associated with resistance to doxorubicin. Anticancer Res. 1986;6(4): 649-652.

7. Kellen JA. The reversal of multidrug resistance in cancer (review). Anticancer Res. 1993;13(4):959-961.

8. Brigger I, Dubernet C, Couvreur P. Nanoparticles in cancer therapy and diagnosis. Adv Drug Deliv Rev. 2002;54(5):631-651.

9. Slowing II, Vivero-Escoto JL, Wu CW, Lin VS. Mesoporous silica nanoparticles as controlled release drug delivery and gene transfection carriers. Adv Drug Deliv Rev. 2008;60(11):1278-1288.

10. Ariga K, Lvov YM, Kawakami K, Ji Q, Hill JP. Layer-by-layer self-assembled shells for drug delivery. Adv Drug Deliv Rev. 2011;63(9): $762-771$.

11. Kawakami K, Ebara M, Izawa H, Sanchez-Ballester NM, Hill JP, Ariga K. Supramolecular approaches for drug development. Curr Med Chem. 2012;19(15):2388-2398.

12. Lian H-Y, Liang Y-H, Yamauchi Y, Wu KCW. A hierarchical study on load/release kinetics of guest molecules into/from mesoporous silica thin films. J Phys Chem C. 2011;115(4):6581-6590.

13. Torelli-Souza RR, Cavalcante Bastos LA, Nunes HGL, Camara CA, Amorim RVS. Sustained release of an antitumoral drug from alginatechitosan hydrogel beads and its potential use as colonic drug delivery, J Appl Polym Sci. 2012;126:E409-E417.

14. Waki K, Anno K, Ono T, Ide T, Chayama K, Tahara H. Establishment of functional telomerase immortalized human hepatocytes and a hepatic stellate cell line for telomere-targeting anticancer drug development. Cancer Sci. 2010;101(7):1678-1685.

15. Dibb M, Ang YS. Targeting the cell cycle in esophageal adenocarcinoma: an adjunct to anticancer treatment. World J Gastroenterol. 2011;17(16): 2063-2069.

16. Petros RA, DeSimone JM. Strategies in the design of nanoparticles for therapeutic applications. Nat Rev Drug Discov. 2010;9(8):615-627.

17. Rejman J, Oberle V, Zuhorn IS, Hoekstra D. Size-dependent internalization of particles via the pathways of clathrin- and caveolaemediated endocytosis. Biochem J. 2004;377(Pt 1):159-169.

18. Fadeel B, Garcia-Bennett AE. Better safe than sorry: Understanding the toxicological properties of inorganic nanoparticles manufactured for biomedical applications. Adv Drug Deliv Rev. 2010;62(3):362-374.

19. Wu KC-W, Jiang X, Yamauchi Y. New trend on mesoporous films: precise controls of one-dimensional (1D) mesochannels toward innovative applications. J Mater Chem. 2011;21:8934-8939.

20. Wu KC-W, Yamauchi Y. Controlling physical features of mesoporous silica nanoparticles (MSNs) for emerging applications. J Mater Chem. 2012;22:1251-1256.

21. Vivero-Escoto JL, Chiang Y-D, Wu KC-W, Yamauchi Y. Recent progress in mesoporous titania materials: adjusting morphology for innovative applications. Sci Technol Adv Mater. 2012;13:013003.

22. Ariga K, Vinu A, Yamauchi Y, Ji Q, Hill JP. Nanoarchitectonics for mesoporous materials. Bull Chem Soc Jpn. 2012;85:1-32.

23. Vivero-Escoto JL, Slowing II, Trewyn BG, Lin VS. Mesoporous silica nanoparticles for intracellular controlled drug delivery. Small. 2010;6(18):1952-1967.
24. George M, Abraham TE. Polyionic hydrocolloids for the intestinal delivery of protein drugs: alginate and chitosan - a review. J Control Release. 2006;114(1):1-14.

25. Tønnesen HH, Karlsen J. Alginate in drug delivery systems. Drug Dev Ind Pharm. 2002;28(6):621-630.

26. Rajesh P, Khuller G. Alginate as a drug delivery carrier. In: Yarema KJ, editor. Handbook of Carbohydrate Engineering. Boca Raton, FL: CRC Press; 2005:799-816.

27. Clark AH, Ross-Murphy SB. Structural and mechanical properties of biopolymer gels. Adv Polym Sci. 1987;83:57-192.

28. Novikova LN, Mosahebi A, Wiberg M, Terenghi G, Kellerth JO, Novikov LN. Alginate hydrogel and matrigel as potential cell carriers for neurotransplantation. J Biomed Mater Res A. 2006;77(2):242-252.

29. Tan WH, Takeuchi S. Monodisperse alginate hydrogel microbeads for cell encapsulation. Adv Mater. 2007;19:2696-2701.

30. Knill CJ, Kennedy JF, Mistry J, et al. Alginate fibres modified with unhydrolysed and hydrolysed chitosans for wound dressings. Carbohydr Polym. 2004;55:65-76.

31. West TP, Preece JE. Bulk alginate encapsulation of Hibiscus moscheutos nodal segments. Plant Cell Tissue Organ Cult. 2009;97:345-351.

32. Srivastava R, McShane MJ. Application of self-assembled ultra-thin film coatings to stabilize macromolecule encapsulation in alginate microspheres. J Microencapsul. 2005;22(4):397-411.

33. Ribeiro CC, Barrias CC, Barbosa MA. Calcium phosphate-alginate microspheres as enzyme delivery matrices. Biomaterials. 2004;25(18): 4363-4373.

34. Zhu H, Srivastava R, Brown JQ, McShane MJ. Combined physical and chemical immobilization of glucose oxidase in alginate microspheres improves stability of encapsulation and activity. Bioconjug Chem. 2005;16(6):1451-1458.

35. Joshi A, Keerthiprasad R, Jayant RD, Srivastava R. Nano-in-micro alginate based hybrid particles. Carbohydr Polym. 2010;81:790-798.

36. Motwani SK, Chopra S, Talegaonkar S, Kohli K, Ahmad FJ, Khar RK. Chitosan-sodium alginate nanoparticles as submicroscopic reservoirs for ocular delivery: formulation, optimisation and in vitro characterisation. Eur J Pharm Biopharm. 2008;68(3):513-525.

37. $\mathrm{Hu}$ Y, Hu N. pH-dependent behaviors of electroactive myoglobin loaded into layer-by-layer films assembled with alginate and hydroxyethyl cellulose ethoxylate. J Phys Chem B. 2008;112(31):9523-9531.

38. Chan E-S, Lim T-K, Ravindra P, Mansa RF, Islam A. The effect of low air-to-liquid mass flow rate ratios on the size, size distribution and shape of calcium alginate particles produced using the atomization method. J Food Eng. 2012;108:297-303.

39. Liang HF, Chen SC, Chen MC, Lee PW, Chen CT, Sung HW. Paclitaxelloaded poly(gamma-glutamic acid)-poly(lactide) nanoparticles as a targeted drug delivery system against cultured HepG2 cells. Bioconjug Chem. 2006;17(2):291-299.

40. Guo H, Lai Q, Wang W, et al. Functional alginate nanoparticles for efficient intracellular release of doxorubicin and hepatoma carcinoma cell targeting therapy. Int J Pharm. 2013;451(1-2):1-11.

41. Iancu C, Mocan L, Bele C, et al. Enhanced laser thermal ablation for the in vitro treatment of liver cancer by specific delivery of multiwalled carbon nanotubes functionalized with human serum albumin. Int $J$ Nanomedicine. 2011;6:129-141.

42. Liao YT, Wu KC, Yu J. Synthesis of mesoporous silica nanoparticleencapsulated alginate microparticles for sustained release and targeting therapy. J Biomed Mater Res B Appl Biomater. 2014;102(2): 293-302.

43. Arosio D, Manzoni L, Araldi EM, Caprini A, Monferini E, Scolastico C. Functionalized cyclic RGD peptidomimetics: conjugable ligands for $\alpha v \beta 3$ receptor imaging. Bioconjug Chem. 2009;20(8):1611-1617.

44. Chang RHY, Jang J, Wu KC-W. Cellulase immobilized mesoporous silica nanocatalysts for efficient cellulose-to-glucose conversion. Green Chem. 2011;13:2844-2850.

45. Herrero EP, Martín Del Valle EM, Galán MA. Development of a new technology for the production of microcapsules based in atomization processes. Chem Eng J. 2006;117:137-142. 
46. Xu SW, Jiang ZY, Lu Y, Wu H, Yuan WK. Preparation and catalytic properties of novel alginate-silica-dehydrogenase hybrid biocomposite beads. Ind Eng Chem Res. 2006;45:511-517.

47. Morgan AW, Roskov KE, Lin-Gibson S, Kaplan DL, Becker ML, Simon CG Jr. Characterization and optimization of RGD-containing silk blends to support osteoblastic differentiation. Biomaterials. 2008;29(16):2556-2563.

48. Choi KY, Yoon HY, Kim JH, et al. Smart nanocarrier based on PEGylated hyaluronic acid for cancer therapy. ACS Nano. 2011;5(11): 8591-8599.

49. Brandhonneur N, Chevanne F, Vié V, et al. Specific and non-specific phagocytosis of ligand-grafted PLGA microspheres by macrophages. Eur J Pharm Sci. 2009;36(4-5):474-485.
50. Shimoda A, Sawada S, Akiyoshi K. Cell specific peptide-conjugated polysaccharide nanogels for protein delivery. Macromol Biosci. 2011;11(7):882-888.

51. Rosenholm JM, Meinander A, Peuhu E, et al. Targeting of porous hybrid silica nanoparticles to cancer cells. ACS Nano. 2009 27;3(1): 197-206.

52. Tedja R, Lim M, Amal R, Marquis C. Effects of serum adsorption on cellular uptake profile and consequent impact of titanium dioxide nanoparticles on human lung cell lines. ACS Nano. 2012;6(5): 4083-4093. 


\section{Supplementary materials}

A

$\mathrm{N}_{2}$ adsorption and desorption of MSN

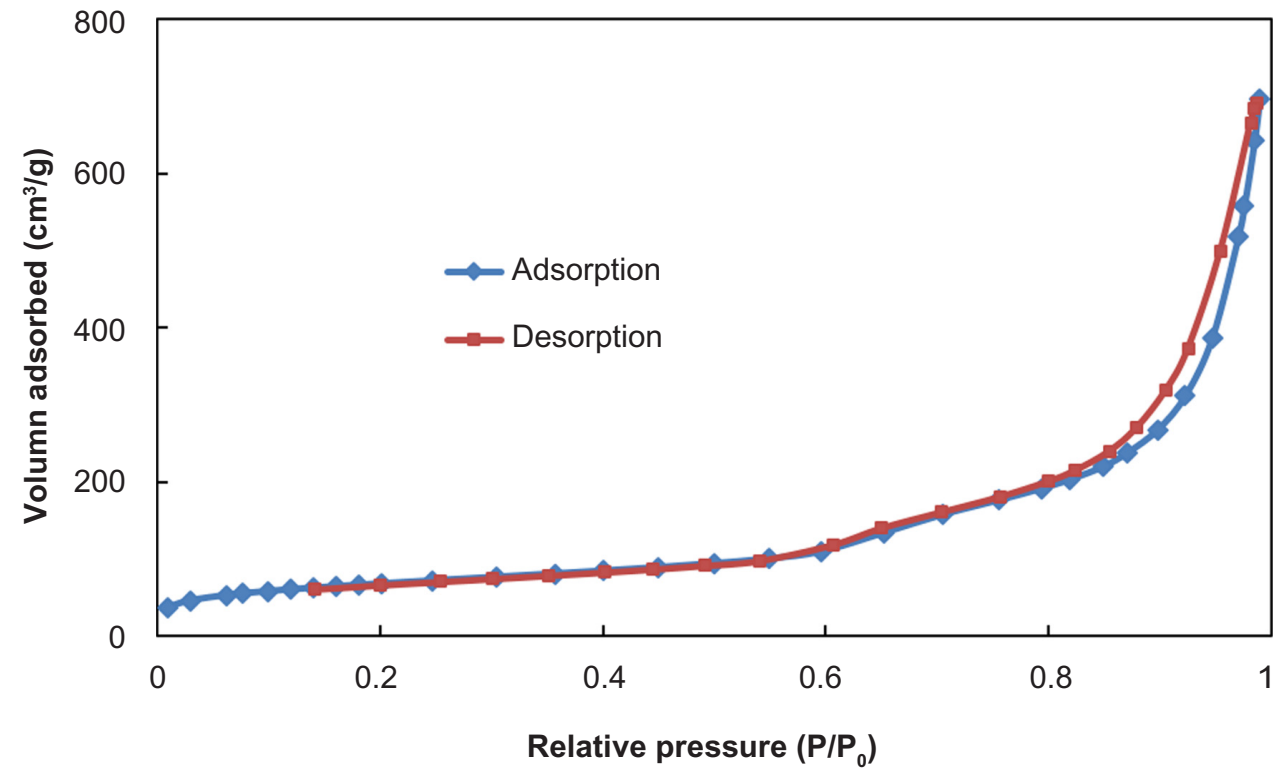

B Pore size distribution of MSN

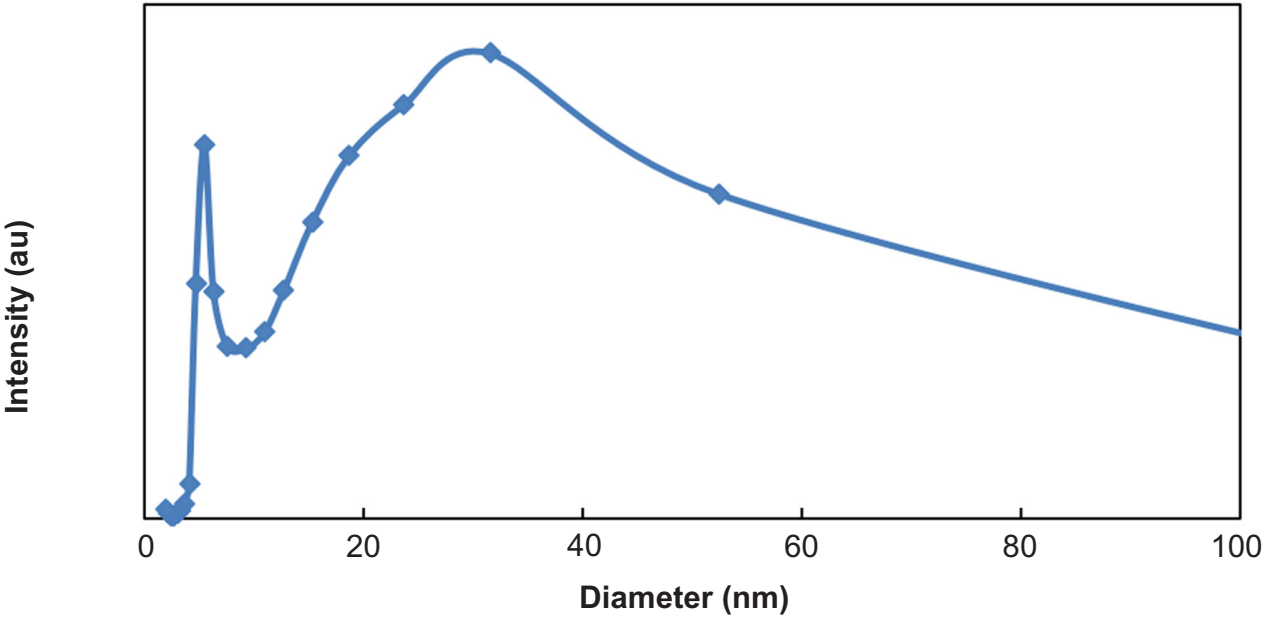

Figure SI (A) $\mathrm{N}_{2}$ adsorption and desorption of mesoporous silica nanoparticles (MSNs). The surface area of MSNs calculated from the BET model was around 285 m²/g. (B) The pore size distribution of MSN. The pore sizes of MSN calculated for the BJH model were 5 and $30 \mathrm{~nm}$, respectively.

Abbreviations: BET, Brunauer-Emmett-Teller; BJH, Barret-Joyner-Halenda.

\section{Publish your work in this journal}

The International Journal of Nanomedicine is an international, peerreviewed journal focusing on the application of nanotechnology in diagnostics, therapeutics, and drug delivery systems throughout the biomedical field. This journal is indexed on PubMed Central, MedLine, CAS, SciSearch $\AA$, Current Contents $\AA /$ Clinical Medicine,
Journal Citation Reports/Science Edition, EMBase, Scopus and the Elsevier Bibliographic databases. The manuscript management system is completely online and includes a very quick and fair peer-review system, which is all easy to use. Visit http://www.dovepress.com/ testimonials.php to read real quotes from published authors. 